\title{
Morphometric study of liver cell nuclei in hepatomas using an interactive computer technique: (i) nuclear size and shape
}

\author{
ROGER JAGOE, CHRISTOPHER SOWTER, SHEILA DANDY, GERARD SLAVIN \\ From Northwick Park Hospital and Clinical Research Centre, Harrow, Middlesex, HAl 3 UJ
}

SUMMARY Liver cells from 20 normal livers and 20 hepatomas have been studied in histological sections using an interactive computer method which measures nuclear size and shape. The variables which gave best discrimination between malignant and benign nuclei were the standard deviations of nuclear shape measurements. Though the liver is an ideal tissue for computer study it is considered that such measurements may act as a model for analysis of nuclei of other tissues.

Nuclear changes are important in the assessment of neoplasia by pathologists. These include changes in size, shape, and texture of nuclei which may be measured objectively by automatic or manually assisted computer methods. Such measurements have been applied recently to uterine carcinomas, ${ }^{1}$ lymphomas and normal lymphoid tissue,${ }^{2-4}$ gastric carcinomas $^{5}$ and bladder tumours ${ }^{6} 7$ either in histological sections or cytological preparations.

In this study we have investigated nuclear size and shape as discriminants of malignant and normal liver cell nuclei in histological sections. The shape of the nucleus is commonly assessed by the relation of nuclear perimeter and area $\left(\mathrm{P}^{2} / 4 \pi \mathrm{A}\right)$ which assesses deviations from circularity. In addition to $\mathrm{P}^{2} / 4 \pi \mathrm{A}$ alterations in shape have been assessed by measurements of changes in the curvature of the nuclear boundary. We have chosen to investigate the liver as an ideal tissue for computer analysis with the expectation that it will serve as a model for other tissues.

\section{Material and methods}

Liver biopsy material was obtained from the files of Northwick Park Hospital and the Clinical Research Centre. Ten normal livers and 10 hepatomas were selected for examination. All the material had been fixed in formol saline and processed by standard procedures to produce $4 \mu \mathrm{m}$ paraffin sections stained by haematoxylin and eosin. The sections were then examined using a Magiscan (Joyce Loebl) Image
Analysis System. Each section was examined with $\times$ 100 oil immersion objective. Maximum nuclear contrast was obtained using a Kodak green filter.

Thirty liver parenchyma cell nuclei were chosen at random in each section. A computer program selected any one of 81 possible fields and a randomly chosen point was displayed within each field. The boundaries of the nearest five liver cell nuclei to the point were delineated and measured. A proportion of nuclei could be automatically detected using grey scale segmentation or edge detection routines but at the magnification chosen it was clear that some nuclei could not be so detected. We therefore chose to define each nuclear membrane interactively by drawing the boundary using the light-pen system. The magnification chosen gave a monitor image of about 1000 square pixels for the smallest nucleus examined. This was large enough to reduce the error of area measurements in repeated manual tracing of a typical nuclear boundary to less than $5 \%$.

A total of nine size and shape measurements or derived values were made on each boundary. The results were used to select those parameters which best differentiated normal from malignant nuclei. Reproducibility was tested by repeating the measurements on half the original sections. Validity of the method was tested by the discriminating ability of the selected parameters on a set of 10 further sections where the diagnosis was unknown to the operator.

SIZE AND SHAPE MEASUREMENTS The area within each nuclear boundary was computed and used as a simple measurement of size. The 
simplest and best known shape measurement perimeter squared divided by $4 \times \pi \times$ area $\left(\mathrm{P}^{2} / 4 \pi \mathrm{A}\right)$ has been used by many workers ${ }^{89}$ and is a component of commercial packages. Deviations of the value from unity reflect deviations from circularity. However there has been criticism of its ability to differentiate dissimilar complex shapes which may produce the same value. ${ }^{9}$ Ideally it is independent of size and orientation but this is not strictly true of shapes which are defined by the intersections of a coordinate grid. Since the perimeter is squared it is important that any error in its estimation be as small as possible. Such error may not be trivial for a shape digitised on a square grid. For example in the worst case the calculation of the length of a digitised straight line segment at an angle of $22.5^{\circ}$ to one of the axes is in error by about $8 \%$. By making the corrections shown in Fig. 1 this error can be reduced to less than $1 \%$. Using this method the $\mathrm{P}^{\mathbf{2}} / 4 \pi \mathrm{A}$ measurements on a square as it was rotated varied by a maximum of $3 \%$.

The measurement $\mathrm{P}^{2} / 4 \pi \mathrm{A}$ was computed on the original traced nuclear boundary. The other shape measurements are size-dependent, and to compensate for this the boundary of each nucleus was expanded about its centre of gravity so that its area was normalised to a size of about 10000 square pixels before shape assessment. A smoothing procedure was applied to even out the small irregularities generated by area normalisation. The new boundary was detected and encoded by a routine which returns a direction-coded boundary list. Fig. 2 shows such a list with elements $P_{i}$ for an arbitrary shape. The perimeter of the normalised area was calculated from this list using the corrections noted above.

Another way of encoding the boundary is to express it as a curvature list with elements $C_{i}$. This is derived from the direction list by the relation:

$$
\mathrm{C}_{\mathrm{i}}=\operatorname{octmod}\left(\mathrm{P}_{\mathrm{i}}-\mathrm{P}_{\mathrm{i}-1}+11\right)-3
$$

where octmod is modulus to the base $8 .^{10}$ For a relatively smooth boundary these values are usually 0 or \pm 1 . A plot of this curvature list does not look very interesting as the main features of the shape are not apparent; however, by a suitable process of smoothing ${ }^{10}$ the inherent information becomes more obvious. The smoothing technique replaced each element by the average of itself plus its nearest eight neighbours. This procedure is repeated four times. The resulting curvature function $f(\mathrm{C})$ for a boundary can be seen in Fig. 3. The curvature function reflects the boundary convexities as peaks and its concavities as troughs.

Six additional measurements of shape were made on the curvature function and were:
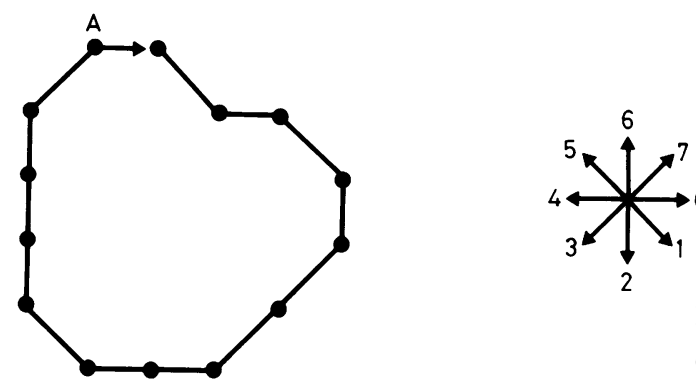

Direction coded boundary list starting clockwise at A $(p 1 p 2 \ldots p 14)=010123344556667$

Curvature coded boundary list

$(c 1 c 2 \ldots . c 14)=1-11111101001100011$

Fig. 2 Direction and curvature lists for an arbitrary shape. The boundary can be coded by starting at an arbitrary grid point, $A$, and recording clockwise the direction of the next grid point. This will be one of 8 directions (0-7) as illustrated. The curvature coded list is obtained from a discrete differentiation of the direction list.

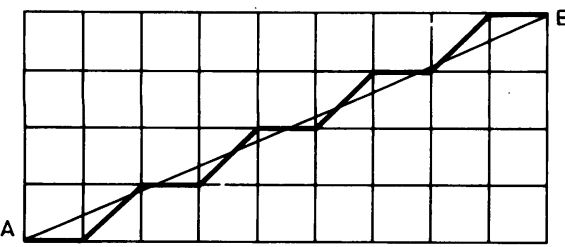

${ }^{B}$ The corrected digital length is calculated by replacing all sections of: Type 1 (a) by $\sqrt{3^{2}+1^{2}}=\sqrt{ } 10$ Type 1 (b) by $\sqrt{3^{2}+2^{2}}=\sqrt{ } 13$

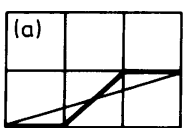

True length of $A B$ Digital length of $A B$ Corrected digital length of $A B=2 \sqrt{ } 10+v 13=9.93$

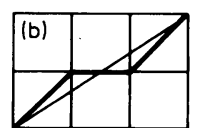

Error

$$
\begin{aligned}
& =\sqrt{9^{2}+L^{2}}=9.85 \\
& =5+4 V 2=10.66+8.2 \%
\end{aligned}
$$

$+0.8 \%$
Fig. 1 Error correction for digital length. If $A B$ is part of $a$ straight line segment of a boundary which has been detected on a digital tracking device it will be defined by the set of grid co-ordinates closest to the line. The digital length is the distance between successive co-ordinates and is generally greater than the true length. This can be partially corrected as illustrated. 


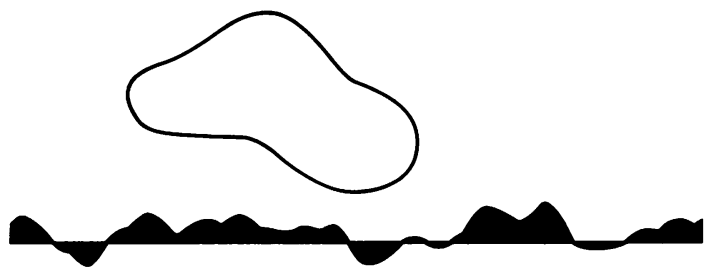

Fig. 3 The curvature function for the boundary of the object, starting at the top and travelling clockwise. The three concavities are represented by the troughs below the axis.

1 Mean value of $f(C)$

2 Standard deviation of $f(\mathrm{C})$

3 Minimum value of $f(C)$

4 Maximum value of $f(C)$

5 Sum of $f^{2}(\mathrm{C})$

6 Range of $f(C)$.

\section{Results}

The size and shape measurements were examined in two ways. In the first instance the results from the nuclei of all the hepatomas were pooled and compared with the pooled results of the non-neoplastic nuclei. This gave two groups of 300 nuclei in each group. The size distributions of the nuclei are shown in Fig. 4. There is considerable overlap between the groups and although the largest cells belong to the hepatomas it is also clear that the smallest nuclei are also from tumours.

The distributions of the $\mathrm{P}^{2} / 4 \pi \mathrm{A}$ shape measurements are shown in Fig. 5. There is large overlap between nuclei from normal livers and hepatomas near to a value of 1 but the values of $\mathrm{P}^{2} / 4 \pi \mathrm{A}$ for the hepatoma nuclei extend considerably further from unity reflecting loss of circularity. Of the nuclei taken from tumours, $27 \%$ have a larger $\mathrm{P}^{2} / 4 \pi \mathrm{A}$ value than the largest value for the nonneoplastic nuclei.

The distributions of the standard deviation of the curvature function of the nuclear boundaries for the two groups is shown in Fig. 6 where it can be seen that the spread of values for the tumour nuclei is much larger- $32 \%$ of the malignant values extend beyond the upper limit of the normal values.

An alternative and more sensible way to interpret the results is to derive measurements from the 30 nuclei selected from each single specimen. There are nine basic measurements on each nucleus. The mean values and standard deviations of each of these measurements were computed giving 18 derived measurements on 20 specimens. All of the derived measurements of shape completely separated hepatomas from the non-neoplastic specimens, whilst the mean and standard deviations of the nuclear
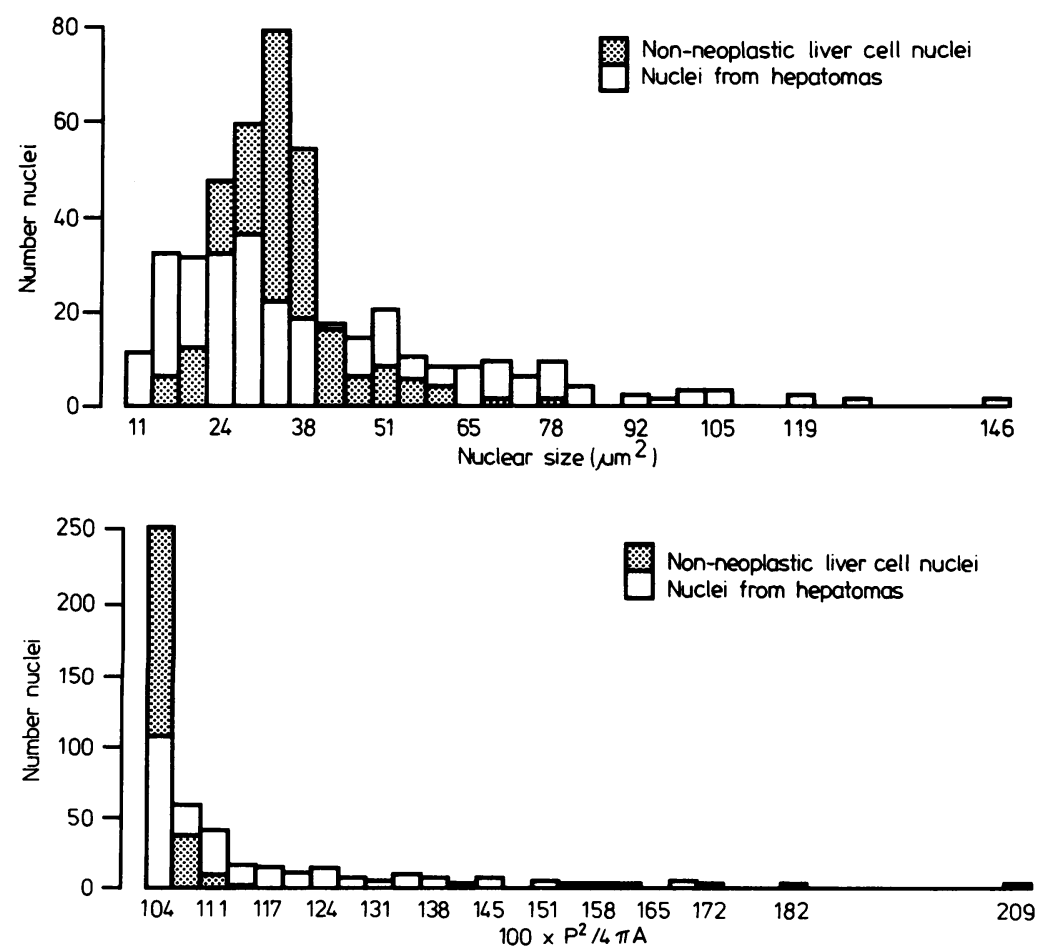

Fig. 4 Comparison of size distributions of nuclei from malignant liver with nuclei from normal liver.

Fig. 5 Comparison of $P^{2} / 4 \pi A$ distributions of nuclei from malignant liver and normal livers. 


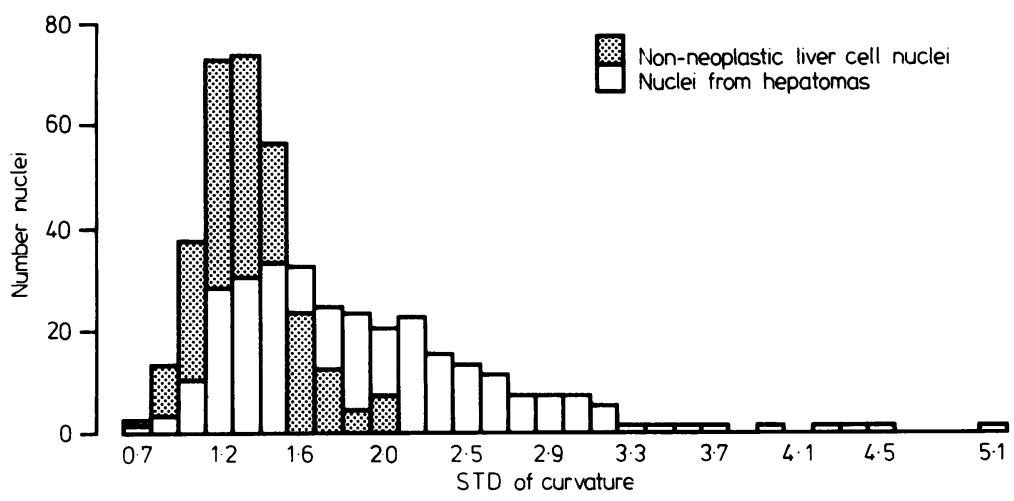

Fig. 6 Comparison of the standard deviation of curvature distributions of nuclear boundaries from malignant and normal livers. areas did not. As an example Fig. 7 shows the mean values of $\mathrm{P}^{2} / 4 \pi \mathrm{A}$ for the 20 sections.

Many of the shape measurements are strongly correlated. The combination of variables which gave the best separation between the hepatomas and nonneoplastic livers was found using a stepwise linear discriminant computer program which maximises the ratio of the difference in means between the groups to the standard deviation within the groups. The variable which gave the best discrimination on its own was the standard deviation of the curvature standard deviation, which is a measure of how much the irregularity of the nuclear boundary varies over a sample of 30 boundaries from a section. The next "best" variable was the standard deviation of the sum of squares of the curvature. This is analogous to the bending energy proposed by Bowie and Young. ${ }^{9}$ A linear combination of these two variables gave a discriminating function which separated nonneoplastic livers from hepatomas as shown in Fig. 9.
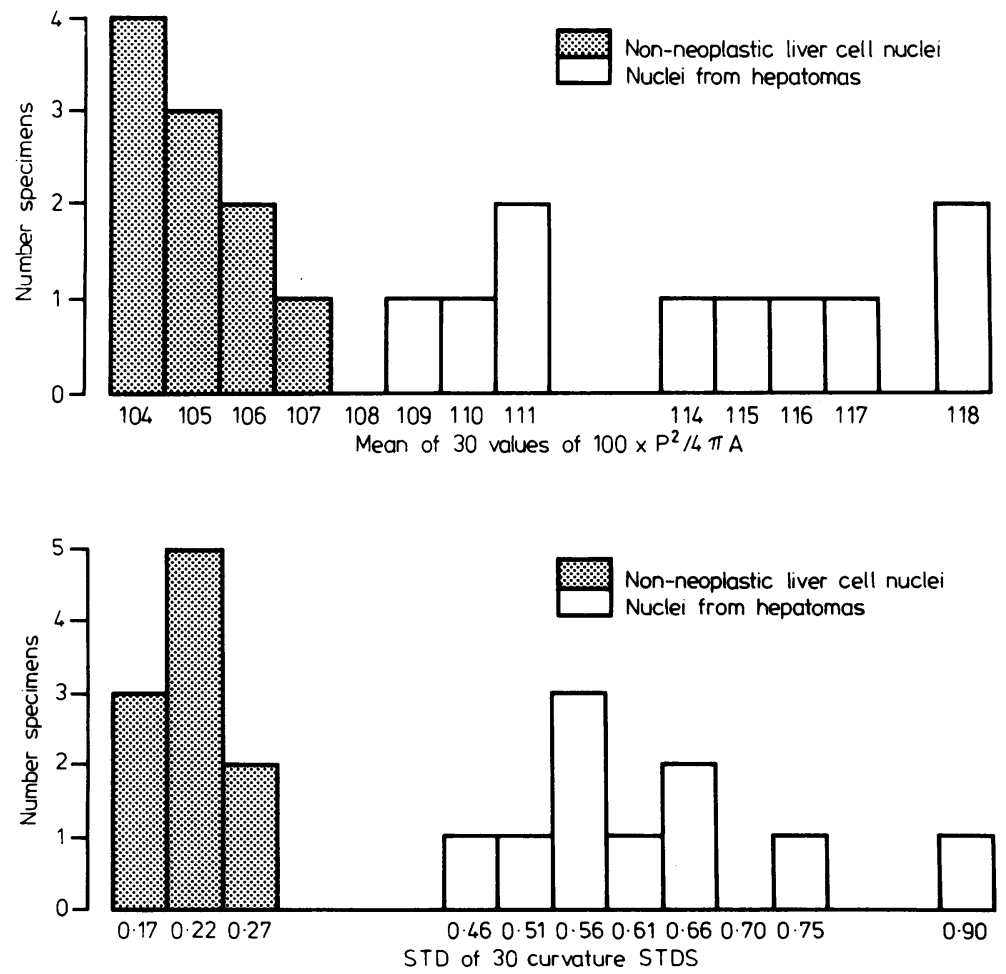

Fig. 7 Mean deviation from circularity as measured by $P^{2} / 4 \pi A$ gives complete separation of benign and malignant biopsy samples.

Fig. 8 There is a sharper separation of samples on the standard deviation of the curvature standard deviation. 


\section{Repeatability}

This was tested by retracing 30 more nuclei on 10 of the previously examined specimens. It is possible, but unlikely, that some of the same nuclei were selected the second time. The discriminant value for each of the repeated specimens was calculated and is shown on the top line of Fig. 9. The connecting lines show how far the repeated values differ from the original ones.

The effectiveness of the discriminant was further tested by applying it to a new set of specimens. Results on these sections are shown on the bottom line of Fig. 9 where it can be seen that nine hepatomas were correctly grouped but that one hepatoma was incorrectly grouped with the nonneoplastic nuclei.

\section{Discussion}

Pathologists use size and shape as well as other nuclear features to distinguish benign from malignant or suspected malignant nuclei. Nuclear pleomorphism may be taken as a measure of differentiation of a tumour. ${ }^{11}$ This study has tried to measure such changes objectively. The liver was selected as an ideal case, for in sections the nucleus is approximately circular and deviations from this shape are easily measured. Moreover the nuclei do not touch and there is little overlap in $4 \mu \mathrm{m}$ sections. In other organs where the nuclei are elongated, oval- or cigar-shaped, then a non-circular shape and the vagaries of section cutting may demand a study of more complicated shape features.

In this study the best separation of benign and malignant nuclei was obtained from the standard deviation of the curvature standard deviation and the next best measurement was the standard deviation of the sum of squares of the curvature. These variables may be combined to give a linear discriminant. However because the sample size is small, too much importance cannot be placed on the exact form of the discriminant and a larger trial could lead to a dif- ferent combination of variables. The important point from this study is that the variables which gave the maximum discrimination are not the mean values of section measurements but rather their standard deviations.

Since the cells in sections from hepatomas are selected at random, measurements may also include some derived from adjacent non-neoplastic cells. In effect there is likely to be sampling from two nuclear populations, neoplastic and non-neoplastic. This explains why the variables which best separate the nuclei are those associated with the spread or standard deviation of measurements on a section. The effect of this dilution of the neoplastic cells will result in a certain amount of variability in the reproducibility of the measurements. It is clear from Fig. 9 that the variability between repeated measurements of the same section was much less than the difference between hepatoma and non-neoplastic sections.

Some care must be exercised with the nuclear sampling, however, to prevent error. The case of the incorrectly grouped hepatoma where a neoplastic liver was grouped with the normals illustrates an example where sampling was inadequate. The sampling technique was programmed to select fields from an area normally covered by a section from a needle biopsy of liver. In this particular case the section was from a much larger open biopsy of which only a relatively small proportion was examined by random fields. Chance determined that only non-neoplastic cells were sampled. When this section was reexamined and the selection program begun in an obviously abnormal area, neoplastic nuclei were included and the measurements then fell into the malignant range.

A further source of variation in manual techniques is likely to arise from the operator either due to increasing skill or increasing fatigue. It is clear that there is a need to replace manual tracing methods by automatic procedures to increase the accuracy, to reduce the tedium, and to increase the speed of the procedure. Unfortunately automatic boundary trac-

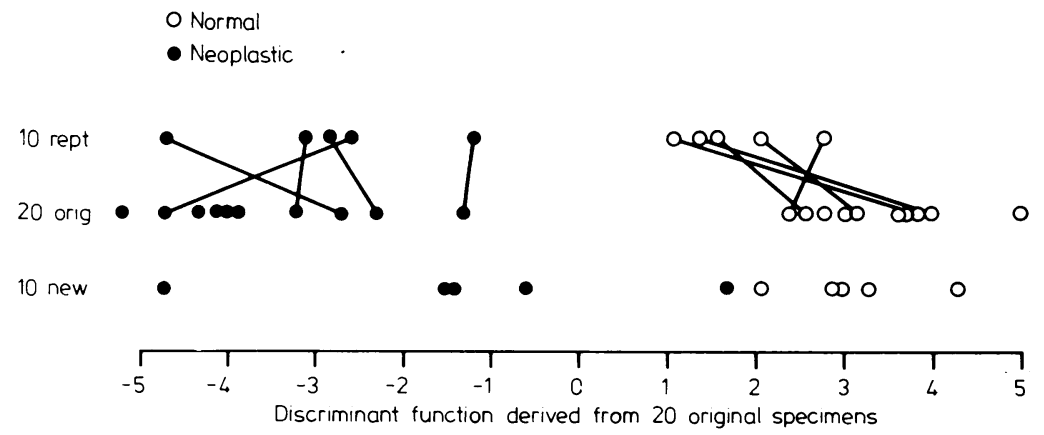

Fig. 9 Separation based on the discriminant function of normal and neoplastic nuclei in original and repeat samples. Note inappropriate coding of one hepatoma as non-neoplastic in the new samples because of sampling error. 
ing routines currently available are only successful in delineating a relatively small proportion of nuclei at high power magnification and this induces a degree of nuclear selection thus biasing results. In a further paper we shall report on comparisons between values achieved by manual and automatic boundary tracing methods.

The development of computer graphics by manual or automatic techniques is a new opportunity for clinical pathologists to apply objective criteria to nuclear features which they normally assess subjectively. The techniques however are relatively new and they need verification in the clinical context. Nuclear size and shape are important measurements in such assessments. In this paper we have attempted to use these parameters to separate normal liver nuclei from those of hepatomas based on collective measurements of thirty randomly selected cells within a single section. With many of the primary measurements there is significant overlap between benign and single neoplastic cells; we have not attempted to establish these. Such definition will require the study of a large series of nuclei and also include nuclei from abnormal but non-neoplastic liver cells-for example, from cirrhosis. However it is clear that with group measurements, clear separation of neoplastic and normal nuclei may be made. We chose to investigate the liver because it seemed an ideal medium for computer analysis with well separated circular nuclei. This study can clearly be extended to include other features such as nuclear texture. These measurements may then be adopted as a model and tested in other tissues, such as gastric or colonic mucosa where the problems for the diag- nostic pathologist are more acute and where objective measurements may be an aid in cases of diagnostic difficulty.

\section{References}

${ }^{1}$ Baak JPA, Diegenbach PC. Quantitative nuclear image $\frac{C}{\sigma}$ analysis:differentiation between normal, hyperplastic, and $\overline{\bar{\omega}}$ malignant appearing uterine glands in a paraffin section. 1. Elementary features for differentiation. Eur J Obstet Gynecol Reprod Biol 1977;7:33-42.

${ }^{2}$ Crocker J, Curran RC. A study of nuclear diameters in lymph node imprints using the Zeiss Microvideomat. J Clin Pathol $\vec{O}$ 1979;32:670-4.

${ }^{3}$ Abbott CR, Blewitt RW, Bird CC. Quantitative analysis of nonHodgkin's lymphoma. J Clin Pathol (in press).

4 Crocker J, Jones EH, Curran RC. A study of nuclear diameters in 용 non-Hodgkin's lymphomas. J Clin Pathol (in press).

5 Boon ME, Kurver PJH, Baak JPA, et al. The application of morphometry in gastric cytological diagnosis. Virchows Arch (Pathol Anat) $1981 ; 393: 159-64$.

${ }^{6}$ Stinson SF, Lilga JC, Reese DH, et al. Quantitation with an automated image analyser of nuclear-cytoplasmic changes induced by hydrocortisone in bladder epithelium. Cancer Res $\mathrm{O}$ 1977;37:1428-31.

${ }^{7}$ Ooms ECM, Essed E, Veldhvizen RW, et al. The prognostic significance of morphometry in T1 bladder tumours. Histopathology 1981;5:311-8.

Bradbury S. Microscopical image analysis: problems and approaches. J Microsc 1979;115:137-50.

${ }^{\circ}$ Bowie JE, Young IT. An analysis technique for biological shape-II. Acta Cytol 1977;21:455-64.

${ }^{10}$ Eccles MJ, McQueen PC, Rosen D. Analysis of the boundaries planar objects. Pattern Recognition 1977;9:31-41.

11 Ptehn RT, Ptehn LM. Pathology of neoplasia. Am J Path 1975;00:529-50.

Requests for reprints to: Mr C Sowter, Histopathology Department, Northwick Park Hospital and Clinical Research Centre, Watford Road, Harrow, Middlesex HA1 3UJ, England. 